\title{
Artigos originais
}

Esta seção destina-se à publicação de artigos enviados espontanea-

mente pelos interessados

\section{Problemas éticos na atenção primária: a visão de especialistas e profissionais}

\section{Ethical problems in primary care: the perspectives of ethicists and professionals}

\section{Luana Torelli da Silva}

Programa de Pós Graduação em Enfermagem da Escola de Enfermagem da Universidade de São Paulo (USP), São Paulo, Brasil.

lutorelli_eeusp@yahoo.com.br

\section{Elma Zoboli}

Departamento de Enfermagem em Saúde Coletiva da Escola de Enfermagem da Universidade de São Paulo (USP), São Paulo, Brasil.

elma@usp.br

Resumo: Trata-se de pesquisa quantitativa e exploratória, com foco na bioética e na atenção básica à saúde. Os objetivos da primeira fase da pesquisa foram identificar problemas éticos vivenciados por enfermeiros e médicos do Programa Saúde da Família (PSF) e verificar a freqüência de sua ocorrência. Na segunda fase, o objetivo foi construir uma espécie de "padrão ouro" com base na visão de um painel de especialistas em ética e bioética e compará-lo com os resultados encontrados na etapa anterior. Para coleta de dados, utilizou-se um questionário auto-aplicado, elaborado com base nos problemas éticos referidos por enfermeiros e médicos em pesquisa anterior de uma das autoras, no que tange as relações com os usuários e famílias, com a equipe multiprofissional e com o Sistema Único de Saúde (SUS). O painel de experts incluiu especialistas em bioética, com formação em enfermagem, medicina, filosofia, teologia e odontologia. De maneira geral, considerando a totalidade dos problemas, os profissionais concordam com o painel em apenas 67,5 \%. O índice de Kappa confirmou tal discordância.

Palavras-chave: Bioética. Saúde da Família. Atenção Primária à Saúde. Humanização da assistência. Ética. 


\title{
Revista Brasileira de Bioética
}

\begin{abstract}
This is a quantitative, exploratory research focused on bioethics and primary care. The objectives of its initial phase were to identify ethical problems experienced by nurses and doctors working within the Family Health Program and observe the frequency of their occurrence. In the second phase, the objective was to build a "golden pattern" based on the opinion of ethicists and bioethicists and to compare this pattern with the results from the first phase. A self-applied questionnaire was used to collect data, based on ethical problems mentioned by doctors and nurses regarding relations between patients and their families and multiprofessional teams and with the health system on previous research by one of the authors. The panel included bioethic specialists professionally trained, such as nurses, medical doctors, philosophers, theologians and dentists. Considering the problems as a whole, professionals and ethicists agreed only in $67.5 \%$ of the situations. The results of Kappa Indices confirmed the discordance between them.
\end{abstract}

Key words: Bioethics. Family health. Primary Healthcare. Humanization of assistance. Ethics.

A atenção à saúde realizada nas unidades básicas de saúde merece lugar nas discussões da bioética, pois problemas do cotidiano perderam espaço para as situações limite, vivenciadas em outros níveis de atenção, que por serem situações dramáticas, que requerem soluções imediatas, são mais freqüentes em tais discussões. A desconsideração em relação às situações cotidianas confere incompletude à abrangência da bioética, pois o foco nos problemas éticos de centros especializados, leva a esquecer que a atenção à saúde não é um conjunto homogêneo de serviços e ações.

Dessa forma, deve-se considerar que os problemas de saúde diferem quanto ao nível das ações e procedimentos oferecidos pelos serviços, assim como os usuários, os familiares e os profissionais de saúde, além dos cenários das instituições que também são diferentes, sem mencionar as distintas soluções possíveis para um mesmo problema. Isto indica que as situações da atenção básica trazem problemas éticos distintos dos vividos na atenção terciária $(1 ; 2 ; 3 ; 4)$.

Pesquisa com enfermeiros e médicos do Programa Saúde da Família (PSF), no município de São Paulo, mostra essas diferenças, a começar pela sutileza e escopo dos problemas éticos vivenciados na atenção básica, que são, em geral, preocupações do cotidiano da 
atenção à saúde e não situações limite, críticas, dramáticas ou dilemáticas. Entretanto, isto não significa que estes problemas sejam menos importantes, mas que a atenção básica lida com fatos e valores distintos; à sua maneira, amplos e complexos, embora de menor apelo dramático (5).

Os encontros com os usuários na atenção básica caracterizam-se por episódios repetidos e aparentemente simples, diferentes das crises bem definidas que pedem pronta solução, típicas da atenção terciária. Por isso, o modo como os problemas éticos emergem na atenção básica pode dificultar seu discernimento (6).

No entanto, a falha em perceber os problemas éticos vividos na atenção primária pode pôr em risco a atenção à saúde prestada nas unidades básicas, rompendo o vínculo estabelecido entre profissionais e usuários. Mesmo que os problemas sejam sutis a ponto de passarem despercebidos, se manejados inadequadamente, podem trazer conseqüências desastrosas para os usuários individualmente, para as famílias, para as relações destes com a equipe de saúde e para a comunidade adscrita (5).

Essas preocupações motivaram o presente estudo, que visou contribuir para o delineamento da interface entre a bioética e a atenção básica, com os objetivos de identificar e comparar os problemas éticos vivenciados por enfermeiros e médicos que atuam no PSF além de verificar a freqüência com que estes profissionais se deparam com problemas éticos.

O presente trabalho retoma parte dos resultados de estudo anterior, com o objetivo de demarcar uma espécie de padrão ouro que pudesse ser usado para uma avaliação mais refinada, por meio da comparação com os resultados previamente encontrados, em especial no que diz respeito à percepção do que é ou não percebido como problema ético. Até então, trabalhava-se apenas com a visão dos próprios atores envolvidos nos cenários que podem gerar problemas éticos, considerando o entendimento que problema ético é tudo aquilo que para alguém é considerado como tal. Assim, se algo for problema ético para alguém, já é problema ético, pois o é pelo menos para a pessoa em questão (7). No entanto, as pesquisadoras sentiram necessidade de consultar especialistas em ética e bioética (doravante denominados "especialistas") para conhecer sua percepção do que 


\section{Revista Brasileira de Bioética}

vem ou não a ser um problema de ordem ética para, assim, poder comparar com os resultados obtidos na pesquisa, realizada apenas com os profissionais.

\section{Método}

Esta pesquisa, financiada com bolsa de iniciação científica da Fundação de Amparo à Pesquisa do Estado de São Paulo (FAPESP), é quantitativa, exploratória, situada no escopo da ética descritiva, enquanto estudo empírico, de cunho não normativo. A ética descritiva investiga factualmente a conduta moral por meio de procedimentos científicos para estudar as atitudes das pessoas $(8 ; 9 ; 10)$. O cenário deste estudo foi formado por unidades básicas de saúde que contam com o PSF no município de São Paulo.

Para efetivação do PSF na capital paulista participam 12 instituições parceiras, que atuam nas diferentes regiões da cidade: Associação Congregação Santa Catarina, Associação Saúde da Família, Associação Comunitária Monte Azul, Casa de Saúde Santa Marcelina, Instituto Adventista de Ensino, Irmandade de Santa Casa de Misericórdia de São Paulo, Fundação Faculdade de Medicina de São Paulo, Organização Santamarense de Educação e Cultura, Universidade Federal de São Paulo, Sociedade Beneficente Israelita Brasileira Albert Einstein, Fundação Euríclides de Jesus Zerbini e Centro de Estudos e Pesquisas Dr. João Amorim (11).

Os sujeitos do estudo foram 24 enfermeiros e 22 médicos de unidades básicas de saúde do município de São Paulo que têm PSF implantado, já que este é uma estratégia preconizada pelo Ministério da Saúde para reorganização da atenção básica no Sistema Único de Saúde (SUS). No total foram incluídos 46 sujeitos, distribuídos pelas diversas instituições parceiras. Este número contempla o tamanho amostral previsto, entre 36 a 72 sujeitos.

As unidades básicas foram selecionadas por sorteio e os profissionais por convite. Para o sorteio, os nomes das unidades, da instituição parceira, com PSF, foram escritos em papéis iguais, colocados em um recipiente e retirados aleatoriamente. Este procedimento foi repetido para cada instituição parceira até completar o universo.

Dos 24 enfermeiros, três (12,5\%) eram do sexo masculino. A mé- 
dia de idade, excluído um sem resposta, foi de 35 anos, com extremos de 24 e 50. O tempo médio de trabalho como enfermeiro era de, aproximadamente, 11 anos e no PSF em torno de dois anos e meio. Três enfermeiros $(12,5 \%)$ trabalhavam no PSF há menos de um ano.

Dos 22 médicos, metade era de cada sexo. O tempo de trabalho como médico era de mais de 13 anos, exceto dois profissionais que trabalhavam há menos de um ano. No PSF, o tempo médio de trabalho era de, aproximadamente, três anos, com quatro médicos registrados há menos de um ano. A idade média era de 36 anos, com extremos de 24 e 60, excluído um, sem resposta.

O projeto foi aprovado pelo Comitê de Ética em Pesquisa da Prefeitura Municipal de São Paulo. A coleta de dados foi feita após autorização das instâncias pertinentes, entre setembro de 2004 e fevereiro de 2005. Para os sujeitos foi pedido o consentimento livre e esclarecido. O instrumento foi um questionário estruturado, auto-aplicado, construído a partir dos problemas éticos apontados por enfermeiros e médicos de equipes de PSF na pesquisa de Zoboli (5). Para cada situação listada foram incluídas opções com base na escala de Likert, categorizadas em "nunca", "comumente" e "ocasionalmente", além da opção "não considero problema ético".

O banco de dados foi feito em Windows Excel 98 e analisado com SPSS for Windows 11.0 (Statistical Package for Social Sciences) e EPIINFO 6.0. Os resultados foram apresentados por meio de números absolutos, médias e proporções, comparadas pelo teste de associação Qui-quadrado, segundo a categoria profissional (enfermeiros e médicos). Nas variáveis em que houve cinco ou menos observações em alguma categoria, utilizou-se o Teste Exato de Fischer. A escolha dos testes para cada situação seguiu recomendação de estatística para o tamanho amostral.

Na extensão do estudo, para a construção do padrão ouro, foram consultados pesquisadores, professores e especialistas da área de bioética, além de presidentes de comissões de ética médica e de enfermagem de serviços de saúde, perfazendo um total de 15 especialistas contatados. Este número foi calculado, considerando que era almejado um painel de especialistas, no mínimo, equivalente a $20 \%$ do total de entrevistados (46), ou seja, entre 9 e 10 participantes. Assim, como é sabido que há perdas, por recusa ou não devolução, decidiu- 


\section{Revista Brasileira de Bioética}

se abordar um número maior para conseguir o mínimo.

O painel incluiu especialistas em bioética, com formação em enfermagem, medicina, filosofia, teologia e odontologia. Esta diversidade levou em conta a multidisciplinaridade que caracteriza a bioética e o fato da pesquisa, na sua primeira fase, não ter demonstrado associação entre a profissão e considerar ou não uma situação como problema ético. Também, por este último motivo, a comparação dos resultados do estudo com a opinião dos especialistas não é apresentada, no presente estudo, de maneira correlacionada com sua formação ou profissão.

Os especialistas foram solicitados, por e-mail ou pessoalmente, a preencherem o questionário auto-aplicado. O formulário foi o mesmo utilizado para os profissionais, excluída a escala de Likert. Ou seja, o especialista tinha apenas de assinalar se, em sua opinião, considerava ou não a situação apresentada na lista como um problema ético. Não foram solicitadas explicações ou justificativas, uma vez que o objetivo era construir um padrão ouro, de natureza quantitativa.

Para avaliar a concordância das opiniões dos dois grupos (profissionais da atenção básica e especialistas em ética e/ou bioética) foi utilizado o índice de Kappa. Este índice estatístico presta-se ao estabelecimento do grau de concordância entre dois avaliadores ao classificarem dois objetos distintos. Cada grupo foi tomado como um avaliador. Foram respondidos nove questionários, o que, garante um número compatível com o mínimo esperado.

\section{Resultados e Discussão}

Cabe lembrar que, para a descrição e discussão dos resultados foram mantidos os três grandes agrupamentos de problemas éticos encontrados na pesquisa de Zoboli (5), cujos resultados serviram de base para o instrumento usado na coleta de dados. Tais agrupamentos incluem os problemas éticos que acontecem nas relações com os usuários e famílias, os que se dão nas relações da equipe e aqueles que ocorrem nas relações com a organização e o SUS. Abaixo, é apresentada a lista com os problemas éticos encontrados em pesquisa realizada por Zoboli (5). 


\section{Problemas éticos levantados pela pesquisa}

1. Dificuldade em estabelecer os limites da relação profissional-usuário;

2. Pré-julgamento dos usuários dos serviços por parte das equipes;

3. Desrespeito do profissional para com o usuário;

4. Indicações clínicas inadequadas;

5. Prescrição de medicamentos que o usuário não poderá comprar;

6. Prescrição de medicamentos mais caros com eficácia igual a dos mais baratos;

7. Solicitação de procedimentos pelo usuário;

8. Como informar ao usuário para conseguir sua adesão ao tratamento;

9. Omissão de informações ao usuário referentes ao seu problema;

10. Acesso dos profissionais de saúde às informações relativas à intimidade da vida familiar e conjugal;

11. Dificuldades para manter a privacidade nos atendimentos domiciliários;

12. Dificuldades para o agente comunitário de saúde para preservar o segredo profissional;

13. Compartilhamento das informações sobre um dos membros da família com os demais;

14. Limites da interferência da equipe no estilo de vida das famílias/usuários;

15. Atitude do médico frente aos valores religiosos próprios e dos usuários;

16. Solicitação de procedimentos por menores de idade sem autorização ou conhecimento dos pais;

17. Recusa dos usuários às indicações médicas;

18. Discussão de detalhes da situação clínica do usuário na sua frente;

19. Falta de compromisso dos profissionais que atuam no PSF com o serviço;

20. Falta de companheirismo e colaboração entre as equipes; 


\section{Revista Brasileira de Bioética}

21. Desrespeito entre os integrantes da equipe;

22. Despreparo dos profissionais para trabalhar no PSF;

23. Dificuldades para delimitar as especificidades e responsabilidades de cada profissional;

24. Omissão dos profissionais frente à indicação clínica imprecisa;

25. Compartilhamento das informações relativas ao usuário e família no âmbito da equipe do PSF;

26. Não solicitação de consentimento da família para relatar sua história em publicação científica;

27. Questionamento da prescrição médica por parte de funcionários da USF;

28. Quebra do sigilo médico por outros membros da equipe ao publicarem relato de casos;

29. Não solicitação de consentimento da equipe para relatar caso em publicação científica;

30. Dificuldades para preservar privacidade por problemas na estrutura física e rotinas da USF;

31. Falta de apoio estrutural para discutir e resolver os problemas éticos;

32. Falta de transparência da direção da USF na resolução de problemas com os profissionais;

33. Excesso de famílias adscritas para cada equipe;

34. Restrição do acesso dos usuários aos serviços;

35. Demérito dos encaminhamentos feitos pelos médicos do PSF;

36. Dificuldades no acesso a exames complementares;

37. Dificuldades quanto ao retorno e confiabilidade dos resultados de exames laboratoriais;

38. Falta de estrutura na USF para a realização de visitas domiciliares;

39. Falta de condições na USF para atendimentos de urgência;

40. Falta de retaguarda de serviço de remoção;

Os resultados primeira fase do estudo foram retomados e serão apresentados de maneira sintética, a fim de explicitar os objetivos atingidos, ou seja, quais os problemas éticos encontrados. Como o propósito neste relato é explicitar os resultados da comparação dos 
achados do estudo com a opinião dos especialistas, será trazido, apenas, o que é ou não problema ético, sem a freqüência de sua ocorrência na prática diária dos profissionais.

Considerando-se as 40 situações geradoras de problemas éticos incluídas na pesquisa, pode-se afirmar de forma resumida que:

- Não houve uma situação que não fosse considerada problema ético, seja no grupo dos enfermeiros ou dos médicos;

- Omissão dos profissionais frente à indicação clínica imprecisa e excesso de famílias adscritas para cada equipe; foram considerados problemas éticos por todos os enfermeiros entrevistados.

- Compartilhamento das informações sobre um dos membros da família com os demais; desrespeito entre os integrantes das equipes; dificuldades para preservar a privacidade por problemas na estrutura física e rotinas da USF; falta de apoio estrutural para discutir e resolver os problemas éticos; e demérito dos encaminhamentos feitos pelos médicos do PSF; foram considerados problemas éticos por todos os médicos entrevistados.

- As seguintes situações foram consideradas problemas éticos pela totalidade dos profissionais entrevistados nas duas categorias: desrespeito do profissional para com o usuário; dificuldade para o agente comunitário de saúde preservar o segredo profissional; questionamento da prescrição médica por parte dos funcionários da USF e quebra do sigilo médico por outros membros da equipe ao publicarem relatos de casos;

- O fato de considerar ou não uma situação apresentada como problema ético, não está relacionado com a categoria profissional, pois os valores de $p$ encontrados não sugerem esta associação;

- Quanto à situação "como informar o usuário para conseguir sua adesão ao tratamento", embora não se tenha demonstrado associação, provavelmente pelo tamanho da amostra, pode-se dizer que há uma tendência à associação, já que o valor de $p$ está muito próximo de 0,05 (foi encontrado $p=0,06$ ).

Na primeira fase do estudo se trabalhou com um entendimento bastante amplo de problema ético. Ou seja, era problema ético qualquer situação que assim fosse tida por pelo menos um dos entrevista- 


\section{Revista Brasileira de Bioética}

dos. Na extensão, como se deseja comparar a visão dos profissionais com a opinião dos especialistas, optou-se por considerar problema ético as situações que tivessem assim sido interpretadas por mais de $50 \%$ de ambas categorias.

Esta opção levou em conta a experiência de uma das autoras em comitês de ética em pesquisa e bioética. Esses colegiados, geralmente, deliberam por consenso, mas em caso de impossibilidade de atingi-lo, a decisão se dará por votação, sendo vencedora a proposta que tiver 50\% mais um dos votos. É válido notar que, se fosse adotado o critério de maioria absoluta, ou seja, mais de $2 / 3$ de respostas positivas, o cenário não seria alterado.

No painel de especialistas, destacam-se as situações que foram consideradas problemas éticos pela totalidade dos consultados: quebra do sigilo médico por outros membros da equipe ao publicarem relato de caso; falta de limites da interferência da equipe no estilo de vida das famílias ou usuários; e atitude do médico frente aos valores religiosos próprios do usuário.

O contrário também merece atenção. Há problemas que não foram considerados como tal pela totalidade do painel: falta de estrutura da USF para realização de visitas domiciliares; falta de condições na USF para atendimento de urgência; e falta de retaguarda de serviço de remoção.

Tomando-se os agrupamentos, a concordância entre especialistas e profissionais varia para cada um. No que diz respeito aos problemas nas relações com o usuário, observa-se a maior proporção de concordância, com somente dois problemas não sendo considerado como tal pelo painel. Isto significa que os grupos concordaram em, aproximadamente, 89\% das situações.

O agrupamento de maior discordância foi o que tratou das relações com a organização e o sistema de saúde. Neste grupo, do total de 11 situações, não houve concordância para 9 delas, ou seja, nestas, $81,82 \%$ dos profissionais consideraram problema ético e os especialistas não. Isto pode ser indicativo de como é difícil separar, no cotidiano, a ética das organizações, da ética na assistência, quando se está na área da saúde. Para os profissionais que vêem suas ações assistenciais comprometidas pelos efeitos das decisões e falhas administrativas e gerenciais, torna-se difícil separar estas dos primeiros, 
no equacionamento ético. O distanciamento dos especialistas parece favorecer esta separação.

Nas relações com a equipe, também há dois problemas para os quais não houve concordância de opinião, com os profissionais respondendo sim e os especialistas não. Como o total deste grupo soma 11 problemas, a discordância aconteceu em 18,18\% das situações.

De maneira geral, ou seja, considerando a totalidade dos problemas, os profissionais concordam com o painel em apenas $67,5 \%$. Em outras palavras, 27 das 40 situações apresentadas são tidas como problemas éticos para os dois grupos. Para análise dos resultados, foram feitos cálculos de valores para o índice de Kappa, considerando dois cenários: um que tomava como sim (é problema ético) quando mais de $50 \%$ do grupo dava respostas positivas e outro assumindo a maioria absoluta, ou seja, era sim quando isto ocorria para mais de $66,67 \%$. Nos dois cenários o índice de Kappa foi zero, o que indica discordância total.

Isto poderia ser explicado por alguns motivos discutidos na introdução do presente artigo e que serviram de motivação para o estudo da ética na atenção básica. É muito difícil encontrar especialistas em bioética e ética que se dediquem ou atuem em atenção básica. Eles estão inseridos em centros de atenção terciária à saúde, podendo ter se baseado nas situações néon destes tipos de serviços para responder o questionário. Os que não são profissionais de saúde, filósofos ou teólogos, podem ter tomado por base a literatura disponível e, neste sentido, há de se considerar que esta tende a repetir o viés que concentra os problemas na área terciária, e faltando reflexão sobre a atenção primária ou básica.

Vale considerar ainda as limitações de um estudo exploratório que não trabalhou com amostras pareadas em tamanho, ou seja, há um número maior de profissionais que especialistas no painel. Foi ponderada a necessidade de aumentar o painel de especialistas, bem como tomar amostra representativa dos profissionais de PSF, o que está sendo desenvolvido no estudo de mestrado de uma das autoras. 


\section{Considerações finais}

Trata-se de estudo quantitativo, exploratório, situado no campo da ética descritiva, cujo objetivo, na extensão, era demarcar um "tipo de padrão ouro" para comparar os achados da primeira etapa do estudo. Embora haja limitações próprias dos estudos exploratórios, os resultados indicam que há uma tendência à discordância entre especialistas e profissionais. Isto deve ser tomado como um alerta para quem trabalha com bioética, já que esta nasce da necessidade de aproximar a academia do serviço nas discussões de ética em saúde. Para quem atua em educação continuada serve também este alerta; talvez seja melhor iniciar as discussões e capacitações pelos problemas mais significativos para os profissionais que estão na prática e não pelo que indica a expertise dos especialistas. Vencida a primeira etapa, reflexão do que faz sentido para os profissionais, poder-se-ia passar para um alargamento das questões pensadas.

Para este estudo, partiu-se do entendimento de que problema ético é qualquer situação tida como tal para alguém. Como pressuposto desta compreensão há o reconhecimento da pluralidade de horizontes éticos. Ao buscar demarcar um padrão ouro com o painel de especialistas, as autoras depararam-se com horizontes éticos distintos, como indica a concordância de apenas 67,5\% das opiniões. Considera-se que, mesmo com as limitações discutidas, foi possível demarcar um tipo de padrão ouro, em vista da concordância entre os especialistas consultados.

Ao se utilizar este padrão para comparar os resultados compilados dos profissionais avaliou-se que os achados apontam para a inevitável pluralidade ética e moral da sociedade de hoje, que se reflete na área da saúde. Como indica Cortina, essa situação também, aponta para a complexidade e necessidade de identificar os deveres morais básicos que deveriam reger a vida das pessoas para uma convivência justa e pacífica, dado o pluralismo (12). Nas equipes de saúde, estes deveres poderão ser definidos tendo como eixo as finalidades do trabalho em saúde e as especificidades de cada serviço. A discordância entre profissionais e especialistas, no que diz respeito à sensibilidade do olhar clínico para problemas éticos, indica a necessidade de traçar pontes entre estes dois universos. E isto somente será possível se os relacionamentos estiverem pautados por uma ética dialógica. 


\section{Referências}

1. Brody H. Transparency: informed consent in primary care. Hasting Centers Report sep/oct, 1989. pp. 5-9.

2. Gracia D. Bioética clínica. Santa Fé de Bogotá: El Buho, 1998.

3. Fetters, MD \& Brody, H. The epidemiology of bioethics. The Journal of Clinical Ethics 1999; 10:107-15.

4. Mayer-Braunack AJ. What makes a problem an ethical problem? An empirical perspective on the nature of ethical problems in general practice. Journal of Medical Ethics 2001; 27:98-103.

5. Zoboli ELCP. Bioética e atenção básica: um estudo de ética descritiva com enfermeiros e médicos do Programa Saúde da Família. [Tese de Doutorado]. São Paulo:Faculdade de Saúde Pública da Universidade de São Paulo; 2003. 6. Sugarman J (org.). Ethics in primary care. New York: McGraw-Hill; 2000, p. xiii-xvi.

7. Gracia D. Procedimentos de decisión en ética clínica. Madrid: Eudema, 1991.

8. Maliandi R. Ética: conceptos y problemas. Buenos Aires: Biblos; 1991.

9. Beauchamp TL \& Childress JF. Principles of biomedical ethics. $5^{\text {a }}$ ed. New York: Oxford University Press; 2001.

10. Sulmasy DP \& Sugarman J. The many methods of medical ethics. In: Sugarman J \& Sulmasy DP. (orgs.) Methods in medical ethics. Washington: Georgetown University Press; 2001, pp. 3-18.

11. Secretaria de Saúde da Prefeitura Municipal de São Paulo. Implantando o PSF no município de São Paulo: balanço de um ano. São Paulo: 2002.

11. Sánchez-González M. La investigación bioética: tipos, funciones y relaciones mutuas. Cuadernos del Programa Regional de Bioética 1998; 6:57-76. 12. Cortina A \& Martinez E. Ética. São Paulo: Edições Loyola; 2005.

Recebido em 03/10/2006 Aprovado em 15/01/2007 